\title{
ENT manifestations of Granulomatosis with polyangiitis
}

\author{
Mohamed Tharwat Hegazy ${ }^{1,2}$, Hamdy Ahmed ${ }^{3}$, Ahmad Mohamed Eltelety ${ }^{4}$ \\ IInternal Medicine Department, Rheumatology, Clinical Immunology Unit, Faculty of Medicine, Cairo University, Cairo 11562, \\ Egypt. \\ ${ }^{2}$ Internal Medicine Department, Rheumatology, Clinical Immunology Unit, Newgiza University, Giza 12256, Egypt. \\ ${ }^{3}$ Department of Medicine, Division of Rheumatology at University of South Alabama, AL 36617, USA. \\ ${ }^{4}$ Otolaryngology Department, AI Kasr Al Ainy School of Medicine, Cairo University, Cairo 11562, Egypt.
}

Correspondence to: Dr. Mohamed Tharwat Hegazy, MD, Internal Medicine Department, Rheumatology, Clinical Immunology Unit, Faculty of Medicine, Cairo University, Al Kasr Al Ainy street, El Manial, Cairo 11562, Egypt. E-mail:

dr_moh_tharwat@kasralainy.edu.eg

How to cite this article: Hegazy MT, Ahmed H, Eltelety AM. ENT manifestations of Granulomatosis with polyangiitis. Vessel Plus 2021;5:46. https://dx.doi.org/10.20517/2574-1209.2021.52

Received: 29 Mar 2021 First Decision: 17 May2021 Revised: 24 May 2021 Accepted: 3 Jun 2021 First online: 15 Jun 2021

Academic Editor: Luca Quartuccio Copy Editor: Yue-Yue Zhang Production Editor: Yue-Yue Zhang

\begin{abstract}
Ear, nose, and throat (ENT) symptoms represent the most frequent manifestations at the onset of granulomatosis with polyangiitis (GPA). The diagnosis of the localized form of GPA remains challenging thus contributing to the delay at the beginning of treatment. This could lead to major sequelae due to tissue destruction. Otolaryngologists play a key role in collaboration with rheumatologists in early GPA diagnosis and treatment. Clinicians should maintain a high index of suspicion and proceed to a more comprehensive evaluation in cases of imperfect response to treatment or with unexplained atypical findings. The challenging issue in localized organ involvement during GPA is to determine whether the non-specific local upper aerodigestive symptoms are caused by a specific or a non-specific inflammatory process. The role of surgery becomes crucial in cases of laryngeal involvement. Lifethreatening airway compromise necessitates urgent surgical intervention. There is an emerging role in the local ENT therapies.
\end{abstract}

Keywords: ENT, granulomatosis with polyangiitis, vasculitis, local therapies 


\section{INTRODUCTION}

Granulomatosis with polyangiitis (GPA) is a necrotizing granulomatous inflammation with necrotizing vasculitis that involves small- and medium-sized blood vessels. It is one of the anti-neutrophil cytoplasmic antibody (ANCA)-associated vasculitides that commonly involves the upper and lower respiratory tract and the kidneys. Ear, nose, and throat (ENT) symptoms represent the most frequent manifestations at the onset of GPA. Their presence can also occur in a milder GPA subset with a better outcome regarding lower renal involvement and lower mortality rate, irrespective of the ANCA status. They can also, not infrequently, coexist with lung nodules ${ }^{[1]}$.

The burden of diagnosing a limited disease with localized manifestations in the upper aerodigestive tract rests mainly with the otolaryngologist. The persistence of sino-nasal symptoms and inadequate response to the standard of care should prompt surgeons to consider a spectrum of diseases including GPA. Thus, the diagnosis of the limited form of GPA is often challenging leading to a delay in the start of treatment. This could lead to major sequelae due to tissue destruction ${ }^{[2]}$.

ENT surgeons play a key role in early GPA diagnosis and treatment in collaboration with rheumatologists, pulmonologists, and nephrologists if needed, especially in GPA cases with negative ANCA antibodies, the diagnosis of which is often more challenging and delayed ${ }^{[3]}$. Limited forms of GPA predominantly affect the upper respiratory tract, whereas generalized forms mostly include lower respiratory tract, kidney, and other systems $s^{[4]}$.

\section{Clinical picture}

Approximately $63 \%$ of GPA patients present with rhinogenic, otologic, or laryngopharyngeal manifestations. Individually, rhinogenic, otologic, and laryngopharyngeal manifestations represent $41 \%$, $16 \%$, and $6 \%$ of GPA manifestations, respectively ${ }^{[5]}$. Of GPA patients undergoing ENT evaluation, $89 \%$ have sino-nasal involvement ${ }^{[6]}$. Clinical presentation is broad and includes an array of non-specific symptoms that embrace, though are not limited to, the following ${ }^{[1,7-15]}$ :

- Symptoms of chronic sinusitis are the most common initial complaint in GPA. Sino-nasal involvement in GPA is often misdiagnosed as chronic sinusitis or rhinitis that fails to respond to the initial conventional therapy.

- Bloody nasal discharge with crusting.

- Sino-nasal masses.

- Oral cavity: purplish hyperplastic gingival lesions, teeth loosening, and failure of oral wounds to heal.

- Strawberry gingival hyperplasia.

- Deformities as a result of bony destruction such as septal perforation (mainly posterior) and saddle nose deformity, which may develop from the involvement of Haversian canals.

- Stridor, possibly leading to respiratory compromise, from tracheal or subglottic granulomatous masses. The sub-glottis is the most affected laryngeal subunit. Between $10 \%$ to $20 \%$ of GPA patients acquire subglottic stenosis ${ }^{[16]}$. Extension of inflammation to the glottis leads to the development of dysphonia. Also, hemoptysis, dyspnea, stridor, and wheezing are possible presentations ${ }^{[17]}$. 
- Otitis media and hearing loss: the middle ear cleft and the mastoid process are the most common sites involved with GPA within the temporal bone. All kinds of hearing loss can result from GPA. Involvement of the Eustachian tube can result in otitis media with effusion leading to conductive hearing loss. This may further predispose to serous otitis media, chronic suppurative otitis media with persistently draining ears, and worsening of hearing loss. The granulomas may present as a mass within the middle ear cavity. Extension of involvement to the inner ear structures can lead to sensorineural hearing loss or mixed hearing loss if combined with conductive hearing loss. Vestibular dysfunction may also result from inner ear affection.

- Lower motor neuron paralysis of the facial nerve may occur if the necrotizing vasculitis involves the neural vasa vasorum.

\section{Investigations}

ENT clinical evaluation using nasal endoscopic assessment can reveal the presence of nasal mucosal crusting, beading, granulation tissue, ulceration, septal perforation (especially posterior), mucosal hyperemia, friability, bloody rhinorrhea, and sinusitis [Figure 1]. Otological assessment using pure tone audiometry, tympanometry, and acoustic reflex testing to evaluate possible sensorineural hearing loss or conductive hearing loss may also be warranted. Involvement of the inner ear may indicate video nystagmography. Pharyngolaryngoscopy is deemed necessary to evaluate for mucosal lesions, allow for biopsy of suspicious masses, determine the presence of airway narrowing and assess the vocal fold mobility. In case of pharyngeal involvement, functional endoscopic evaluation of swallowing can be performed to assess the swallowing ability ${ }^{[1]}$.

\section{Laboratory investigations}

Up to $90 \%$ of patients with generalized GPA have a positive $\mathrm{ANCA}^{[18]}$. Up to $80 \%-90 \%$ of patients with GPA are likely to have C-ANCA directed against proteinase 3 protein in the cytoplasm ${ }^{[19]}$. However, only $60 \%$ of patients with limited GPA, defined as GPA disease isolated to upper or lower respiratory tract with no renal involvement ${ }^{[4]}$, have a positive ANCA test ${ }^{[20]}$. There is growing evidence that ANCA plays a major role in the pathogenesis of GPA. ANCA antibodies play a role in the attachment of neutrophils to the vascular endothelium early in the pathogenesis of the disease ${ }^{[21]}$. Non-specific laboratory tests include complete blood count which may show leukocytosis, eosinophilia, and thrombocytosis, and inflammatory markers such as sedimentation rate and C-reactive protein which can be elevated. Laboratory tests may also show involvement of other organs such as impaired kidney function and abnormal urine analysis ${ }^{[22]}$.

\section{Radiology}

Computerized tomography (CT) of the paranasal sinuses may reveal mucosal thickening, nasal septal destruction, sino-nasal mass with or without orbital involvement [Figure 2]. Bony destruction with resultant osteo-neogenesis is not uncommon. Tracheal stenosis can be seen with GPA. CT scan of the neck is mandatory to determine airway narrowing and aid the surgical planning. Chest $\mathrm{x}$-ray may detect associated lung involvement and further CT chest may be requested ${ }^{[1723-25]}$. If upper airway obstruction is suspected, pulmonary function test may be of a value. Flow volume loops may show characteristic inspiratory and expiratory flattening ${ }^{[26,27]}$.

\section{Pathology}

Although biopsy from upper aerodigestive involved sites is easily accessible, it remains unreliable to make the diagnosis. In one retrospective study, which included 126 patients who were clinically diagnosed with GPA, biopsies taken from the nose, paranasal sinuses, larynx, mouth, periorbital tissue, mastoid, and middle ear were reviewed to assess GPA pathology findings in these sites. All three findings typical for GPA 

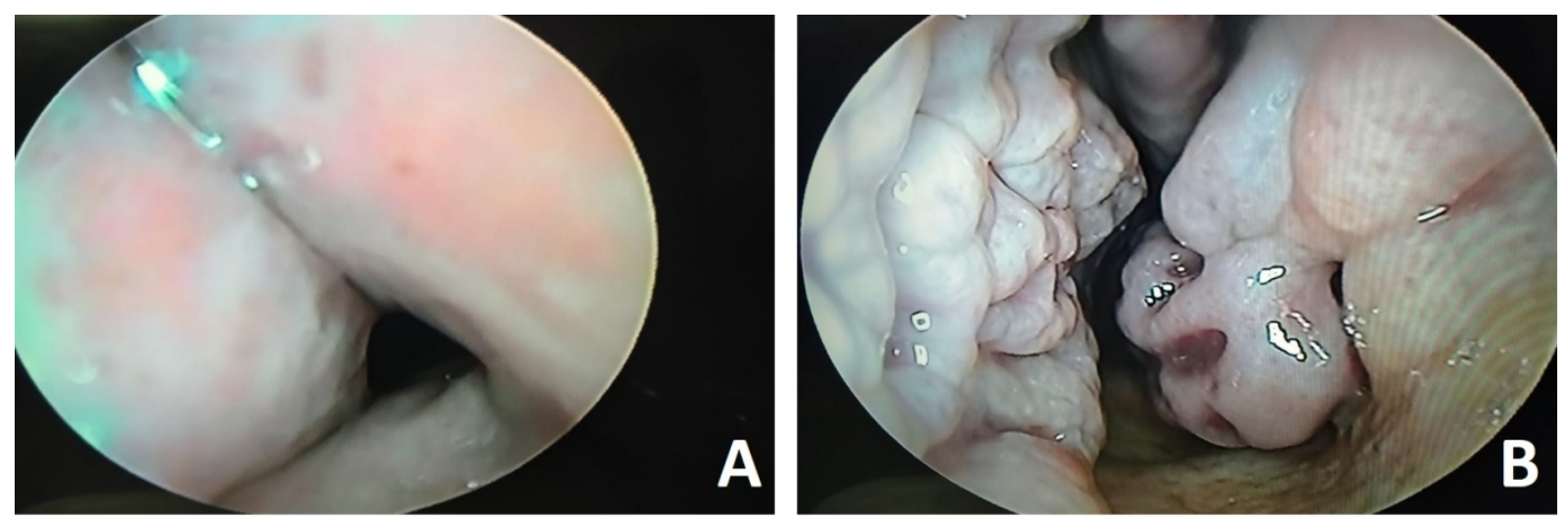

Figure 1. A 55-year-old male previously had bilateral partial inferior turbinectomy for persistent nasal obstruction without improvement after surgery. (A) presents the left nasal vestibule with a visible collapse of the external nasal valve caused by nasal framework disintegration. (B) presents beaded granular nasal mucosa throughout the entire nasal cavity. The patient was referred to a rheumatologist and diagnosed with GPA.

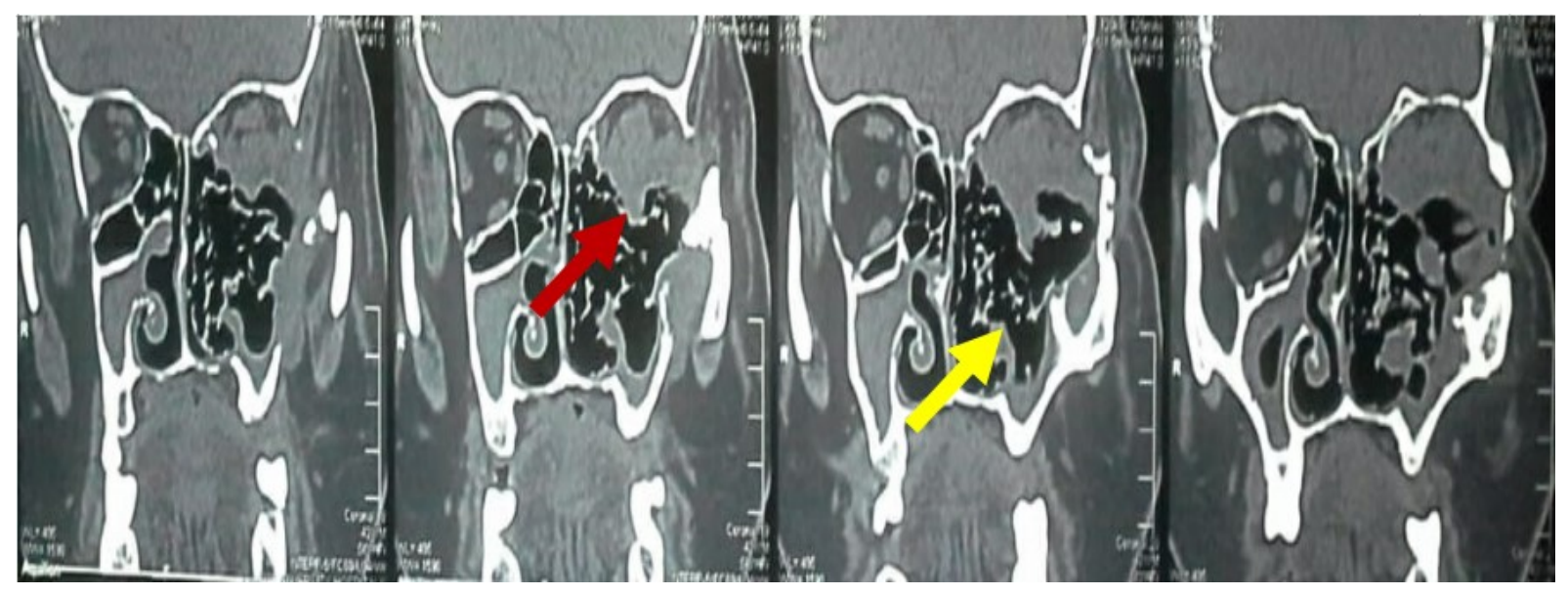

Figure 2. A 39-year-old male patient with a left maxillary mass invading the left orbital contents. There is evident bony destruction with osteoneogenesis. Endoscopic biopsy revealed necrotizing vascular inflammation with extensive necrosis. Red arrow: left nasal mass extending intra-orbitally. Yellow arrow: bone destruction of the medial wall of the left maxilla and medial orbital wall.

including vasculitis, granuloma, and necrosis were found in only $16 \%$ of the patients, while only $21 \%$ had vasculitis and granulomatous changes, and only $23 \%$ had vasculitis and necrosis changes [Figure 3]. Thus, a negative biopsy does not rule out a diagnosis of ANCA vasculitis. However, a biopsy can still be of value in ANCA-negative patients and where other diagnoses, such as malignancy and infection, need to be excluded. In cases of generalized involvement, biopsies from other organs such as kidney and skin are more sensitive ${ }^{[28,29]}$.

\section{Treatment}

A multidisciplinary approach for the management of cases of ANCA-associated vasculitides involving the upper respiratory tract is highly recommended.

\section{Medical treatment}

If there is involvement of a major organ such as lung or kidney or there is life-threatening disease, aggressive treatment should be initiated. For localized disease involving sinuses and the upper airway, a less aggressive approach can be used. Methotrexate is recommended for induction of remission. Mycophenolate 


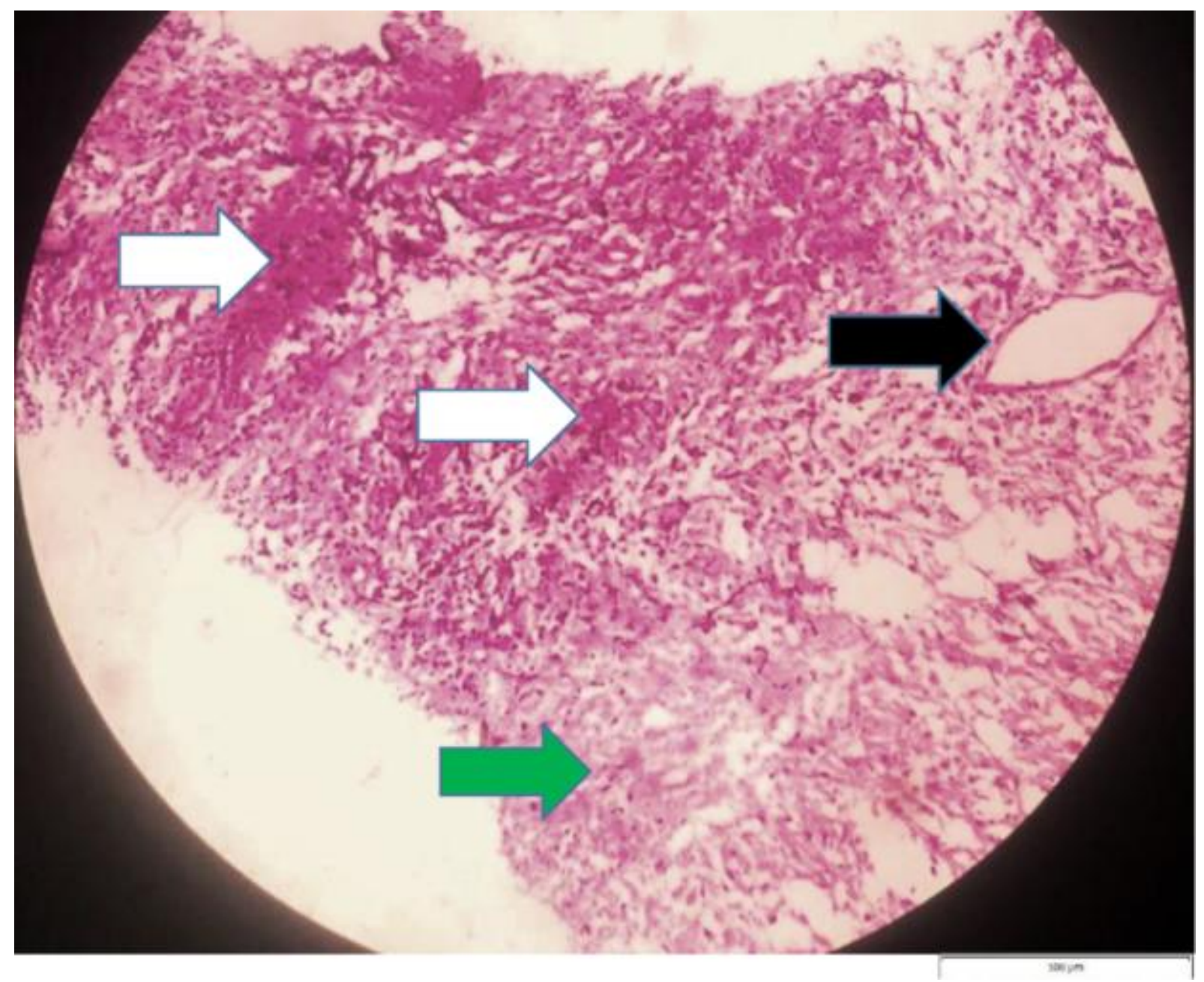

Figure 3. A 34-year-old male patient with a nasal mass. Histology of the nasal mass showing vasculitis (black arrow), granulomatous reaction (white arrows), and areas of necrosis (green arrow) $[\mathrm{H \& E}, \times 200$ (original magnification), scale bar $=100 \mu \mathrm{m}]$. (Courtesy of Dr. Maha Emad Eldein, Lecturer of Pathology, Department of Pathology, Faculty of Medicine, Cairo University, Egypt).

can be used as an alternative. Combination with glucocorticoid can be used in cases of severe disease. If remission is not achieved, cyclophosphamide or rituximab can be used.

For maintenance of remission, methotrexate or azathioprine are recommended. Rituximab can be used as an alternative. Treatment usually continues for two years and sometimes a longer duration may be required $^{[30,31]}$.

\section{ROLE OF LOCAL ENT THERAPY AND COMMON INTERVENTIONS}

Local ENT therapies are part of medical therapy of which immunosuppressive therapy is the keystone in the management of GPA. Actively managing local ENT symptoms does improve the patient's quality of life and - in some cases - is life-saving.

Topical saline nasal irrigations, antibiotic irrigations, and topical nasal steroids may alleviate bothersome sino-nasal symptoms and reduce inflammation. Though being sterile in its origin, GPA can lead to superimposed bacterial infection, and thus, culture-directed antimicrobial therapy may be necessary in 
certain circumstances. Staphylococcus aureus is a common nasal commensal bacteria and is an independent risk factor for relapses in GPA (7-fold relative risk of relapse in chronic nasal carriers). Therefore long-term prophylaxis with sulfamethoxazole and trimethoprim is recommended ${ }^{[32]}$.

The need for endoscopic sinus surgery (ESS) is exceedingly rare. The main role for ESS remains mainly managing complications or intolerable manifestations after exhaustion of all other possible medical therapies. Surgery is extremely difficult given the expected alteration of anatomy by extensive scarring, structural destruction, and previous surgeries.

Sino-nasal GPA can lead to recurrent dacryocystitis, indicating endoscopic dacryocystorhinostomy, mucocele, or pyocele, indicating endoscopic drainage, and/or bone necrosis indicating endoscopic debridement. In cases of saddle nose deformity, rhinoplasty with structural bone grafting can improve the cosmetic and functional outcome after systemic control of disease for at least 12 months ${ }^{[3,343]}$.

On the other hand, the role of surgery is crucial in cases of laryngeal involvement. Life-threatening airway compromise calls for urgent surgical intervention mainly tracheostomy as cricothyroidotomy will not bypass the subglottic obstruction.

Long-term management of airway narrowing utilizes a combination of endoscopic and open surgery. Endoscopic laryngeal dilation with rigid or balloon dilators can achieve a satisfactory outcome. This can be further aided by local injection of intralesional steroids or mitomycin C. Transoral carbon dioxide laser has also been used to resect fibrous bands while ensuring safe hemostasis. Split or augmentation of the cricoid cartilage by open surgery can help widen the narrowed subglottis. On the rare occasion of tracheal involvement solely with mature fibrosis and inactive disease, open resection of affected segment and reanastomosis can lead to successful decannulation ${ }^{[10-12,35]}$.

\section{CONCLUSION}

A multidisciplinary approach for the management of cases with GPA is mandatory for proper early diagnosis and treatment. Although immunosuppressive therapy is the keystone of management of GPA, local therapies do improve the patient's quality of life and - in some cases - are life-saving.

\section{DECLARATIONS}

\section{Authors' contribution}

Wrote and reviewed the manuscript: Hegazy MT, Ahmed H, Eltelety AM

\section{Availability of data and materials}

Not applicable.

\section{Financial support and sponsorship}

None.

\section{Conflict of interests}

All authors declared that there are no conflicts of interest.

\section{Ethical approval and consent to participate}

Not applicable. 


\section{Consent for publication}

Not applicable.

\section{Copyright}

(c) The Author(s) 2021.

\section{REFERENCES}

1. Felicetti M, Cazzador D, Padoan R, et al. Ear, nose and throat involvement in granulomatosis with polyangiitis: how it presents and how it determines disease severity and long-term outcomes. Clin Rheumatol 2018;37:1075-83. DOI PubMed

2. Hernández-Rodríguez J, Hoffman GS, Koening CL. Surgical interventions and local therapy for Wegener's granulomatosis. Curr Opin Rheumatol 2010;22:29-36. DOI PubMed

3. Hauer HA, Bajema IM, van Houwelingen HC, et al; European Vasculitis Study Group (EUVAS). Renal histology in ANCA-associated vasculitis: differences between diagnostic and serologic subgroups. Kidney Int 2002;61:80-9. DOI PubMed

4. JH; Wegener's Granulomatosis Etanercept Trial Research Group. Limited versus severe Wegener's granulomatosis: baseline data on patients in the Wegener's granulomatosis etanercept trial. Arthritis Rheum 2003;48:2299-309. DOI PubMed

5. Srouji IA, Andrews P, Edwards C, Lund VJ. Patterns of presentation and diagnosis of patients with Wegener's granulomatosis: ENT aspects. J Laryngol Otol 2007;121:653-8. DOI PubMed

6. Cannady SB, Batra PS, Koening C, et al. Sinonasal Wegener granulomatosis: a single-institution experience with 120 cases. Laryngoscope 2009;119:757-61. DOI PubMed

7. Fauci AS, Haynes BF, Katz P, Wolff SM. Wegener's granulomatosis: prospective clinical and therapeutic experience with 85 patients for 21 years. Ann Intern Med 1983;98:76-85. DOI PubMed

8. Holle JU, Laudien M, Gross WL. Clinical manifestations and treatment of Wegener's granulomatosis. Rheum Dis Clin North Am 2010;36:507-26. DOI PubMed

9. Alaani A, Hogg RP, Drake Lee AB. Wegener's granulomatosis and subglottic stenosis: management of the airway. J Laryngol Otol 2004;118:786-90. DOI PubMed

10. Polychronopoulos VS, Prakash UB, Golbin JM, Edell ES, Specks U. Airway involvement in Wegener's granulomatosis. Rheum Dis Clin North Am 2007;33:755-75, vi. DOI PubMed

11. Stappaerts I, Van Laer C, Deschepper K, Van de Heyning P, Vermeire P. Endoscopic management of severe subglottic stenosis in Wegener's granulomatosis. Clin Rheumatol 2000;19:315-7. DOI PubMed

12. Nouraei SA, Obholzer R, Ind PW, et al. Results of endoscopic surgery and intralesional steroid therapy for airway compromise due to tracheobronchial Wegener's granulomatosis. Thorax 2008;63:49-52. DOI PubMed

13. Rasmussen N. Management of the ear, nose, and throat manifestations of Wegener granulomatosis: an otorhinolaryngologist's perspective. Curr Opin Rheumatol 2001;13:3-11. DOI PubMed

14. McCaffrey TV, McDonald TJ, Facer GW, DeRemee RA. Otologic manifestations of Wegener's granulomatosis. Otolaryngol Head Neck Surg (1979) 1980;88:586-93. DOI PubMed

15. Santos F, Salviz M, Domond H, Nadol JB. Otopathology of vasculitis in granulomatosis with polyangitis. Otol Neurotol 2015;36:1657-62. DOI PubMed PMC

16. Langford CA, Hoffman GS. Rare diseases.3: Wegener's granulomatosis. Thorax 1999;54:629-37. DOI PubMed PMC

17. Langford CA, Sneller MC, Hallahan CW, et al. Clinical features and therapeutic management of subglottic stenosis in patients with Wegener's granulomatosis. Arthritis Rheum 1996;39:1754-60. DOI PubMed

18. Hoffman GS, Specks U. Antineutrophil cytoplasmic antibodies. Arthritis \& Rheumatism 1998;41:1521-37. DOI PubMed

19. Hagen EC, Daha MR, Hermans J, et al. Diagnostic value of standardized assays for anti-neutrophil cytoplasmic antibodies in idiopathic systemic vasculitis. EC/BCR Project for ANCA Assay Standardization. Kidney Int 1998;53:743-53. DOI PubMed

20. Savige J, Pollock W, Trevisin M. What do antineutrophil cytoplasmic antibodies (ANCA) tell us? Best Pract Res Clin Rheumatol 2005;19:263-76. DOI PubMed

21. Nakazawa D, Masuda S, Tomaru U, Ishizu A. Pathogenesis and therapeutic interventions for ANCA-associated vasculitis. Nat Rev Rheumatol 2019;15:91-101. DOI PubMed

22. Hoffman GS, Kerr GS, Leavitt RY, et al. Wegener granulomatosis: an analysis of 158 patients. Ann Intern Med 1992;116:488-98. DOI PubMed

23. D'Anza B, Langford CA, Sindwani R. Sinonasal imaging findings in granulomatosis with polyangiitis (Wegener granulomatosis): a systematic review. Am J Rhinol Allergy 2017;31:16-21. DOI PubMed

24. Pagnoux C. Updates in ANCA-associated vasculitis. Eur J Rheumatol 2016;3:122-33. DOI PubMed PMC

25. Solans-Laqué R, Bosch-Gil J, Canela M, Lorente J, Pallisa E, Vilardell-Tarrés M. Clinical features and therapeutic management of subglottic stenosis in patients with Wegener's granulomatosis. Lupus 2008;17:832-6. DOI PubMed

26. Girard C, Charles P, Terrier B, et al. Tracheobronchial stenoses in granulomatosis with polyangiitis (Wegener's): a report on 26 cases. Medicine (Baltimore) 2015;94:e1088. DOI PubMed PMC

27. Agrawal A, Sahni S, Marder G, Shah R, Talwar A. Biphasic flow-volume loop in granulomatosis with polyangiitis related unilateral bronchus obstruction. Respir Investig 2016;54:280-3. DOI PubMed

28. Maguchi S, Fukuda S, Takizawa M. Histological findings in biopsies from patients with cytoplasmic-antineutrophil cytoplasmic 
antibody (cANCA)-positive Wegener's granulomatosis. Auris Nasus Larynx 2001;28:S53-8. DOI PubMed

29. Devaney KO, Travis WD, Hoffman G, Leavitt R, Lebovics R, Fauci AS. Interpretation of head and neck biopsies in Wegener's granulomatosis. A pathologic study of 126 biopsies in 70 patients. Am J Surg Pathol 1990;14:555-64. DOI PubMed

30. Yates M, Watts RA, Bajema IM, et al. EULAR/ERA-EDTA recommendations for the management of ANCA-associated vasculitis. Ann Rheum Dis 2016;75:1583-94. DOI PubMed

31. Ntatsaki E, Carruthers D, Chakravarty K, et al; BSR and BHPR Standards; Guidelines and Audit Working Group. BSR and BHPR guideline for the management of adults with ANCA-associated vasculitis. Rheumatology (Oxford) 2014;53:2306-9. DOI PubMed

32. Stegeman CA, Tervaert JW, de Jong PE, Kallenberg CG. Trimethoprim-sulfamethoxazole (co-trimoxazole) for the prevention of relapses of Wegener's granulomatosis. Dutch Co-Trimoxazole Wegener Study Group. N Engl J Med 1996;335:16-20. DOI PubMed

33. Ezzat WH, Compton RA, Basa KC, Levi J. Reconstructive techniques for the saddle nose deformity in granulomatosis with polyangiitis: a systematic review. JAMA Otolaryngol Head Neck Surg 2017;143:507-12. DOI PubMed

34. Coordes A, Loose SM, Hofmann VM, et al. Saddle nose deformity and septal perforation in granulomatosis with polyangiitis. Clin Otolaryngol 2018;43:291-9. DOI PubMed

35. Roediger FC, Orloff LA, Courey MS. Adult subglottic stenosis: management with laser incisions and mitomycin-C. Laryngoscope 2008;118:1542-6. DOI PubMed 